\title{
OPEN Intestinal long non-coding RNAs in response to simulated microgravity stress in Caenorhabditis elegans
}

\author{
Lingmei Sun, Dan Li, Yujie Yuan \& Dayong Wang
}

Long non-coding RNAs (IncRNAs) are important in regulating the response to environmental stresses in organisms. In this study, we used Caenorhabditis elegans as an animal model to determine the functions of intestinal IncRNAs in regulating response to simulated microgravity stress. Among the intestinal IncRNAs, linc-2, linc-46, linc- 61 , and linc- 78 were increased by simulated microgravity treatment, and linc-13, linc-14, linc-50, and linc-125 were decreased by simulated microgravity treatment. Among these 8 intestinal IncRNAs, RNAi knockdown of linc- 2 or linc-61 induced a susceptibility to toxicity of simulated microgravity, whereas RNAi knockdown of linc-13, linc-14, or linc-50 induced a resistance to toxicity of simulated microgravity. In simulated microgravity treated nematodes, linc-50 potentially binds to three transcriptional factors (DAF-16, SKN-1, and HLH-30). RNAi knockdown of daf-16, skn-1, or hlh-30 could suppress resistance of linc-50(RNAi) nematodes to the toxicity of simulated microgravity. Therefore, our results provide an important basis for intestinal IncRNAs, such as the linc-50, in regulating the response to simulated microgravity in nematodes.

During or after spaceflight, some toxic effects on health of humans can be observed due to existence of microgravity and irradiation ${ }^{1-5}$. Nematode Caenorhabditis elegans is highly sensitive to various stresses or toxicants ${ }^{6-10}$. Due to this sensitivity, C. elegans has been frequently used to assess toxic effects of spaceflight on organisms ${ }^{11-14}$. Microgravity during the spaceflight potentially induced toxicity on locomotion behavior, muscle development, and reproduction in nematodes ${ }^{11-14}$. Moreover, microgravity during spaceflight could also induce dysregulation of some genes involved in control of metabolisms, cytoskeletal development, and etc. ${ }^{15-17}$.

Investigations on effects of simulated microgravity can reflect the potential influences of microgravity during spaceflight to a great degree. In nematodes, treatment with simulated microgravity could inhibit locomotion behavior, induce intestinal damage, and activate oxidative stress reflected by reactive oxygen species (ROS) production ${ }^{18-20}$. Meanwhile, simulated microgravity also activated some protective responses, such as mitochondrial unfolded protein response (mt UPR $)^{21}$. More importantly, C. elegans is a powerful animal model for the study of molecular toxicology ${ }^{22}$. In C. elegans, insulin, p38 mitogen-activated protein kinase (MAPK), and Wnt signaling pathways have been identified to be involved in controlling toxicity of simulated microgravity ${ }^{23-25}$. Nevertheless, the molecular basis for response of nematodes to simulated microgravity still remains largely unclear.

Long non-coding RNAs (lncRNAs) are molecules having at least 200 nucleotides. The lncRNAs are a type of noncoding RNAs with many important biological functions ${ }^{26,27}$. In C. elegans, some of lncRNAs have been identified to be required for control of response to environmental toxicants, such as nanopolystyrene and graphene oxide $(\mathrm{GO})^{28,29}$. We assumed that lncRNAs play important functions in regulating response to simulated microgravity. In C. elegans, our previous studies have suggested the crucial function of intestinal barrier in response to simulated microgravity ${ }^{21,23-25}$. In this study, we focused on the identification of intestinal lncRNAs involved in control of response to simulated microgravity. Moreover, we determined the underlying mechanism for linc-50, one of the identified lncRNAs, in regulating response to simulated microgravity.

\section{Results}

Effect of simulated microgravity treatment on ROS production and locomotion behavior in wild-type nematodes. We first investigated the effects of simulated microgravity treatment for 4, 8, 12, or $24 \mathrm{~h}$ on two aspects, induction of ROS production and locomotion behavior. Simulated microgravity treatment for $4 \mathrm{~h}$ did not induce significant ROS production and alteration in locomotion behavior (Fig. 1; Table S1). In 
$\mathrm{a}$ Time (h)
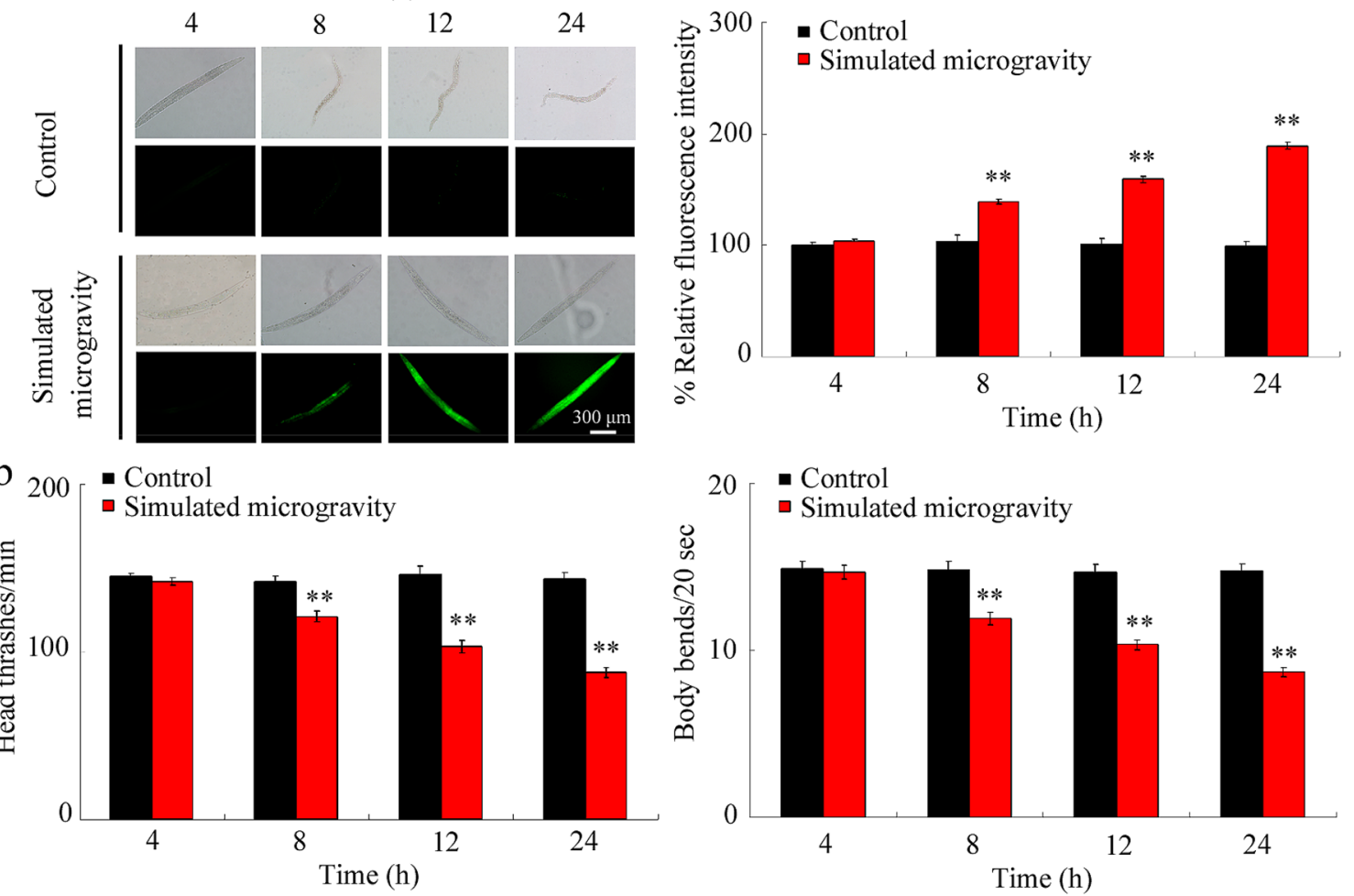

Figure 1. Effects of simulated microgravity treatment on wild-type nematodes. (a) Effects of simulated microgravity treatment on ROS production. $\mathrm{N}=50$. (b) Effects of simulated microgravity treatment on locomotion behavior. $\mathrm{N}=40$. Simulated microgravity treatment was performed in RCCS system at $30 \mathrm{rpm}$ for 4 , 8,12 , or $24 \mathrm{~h}$. Bars represent means $\pm \mathrm{SD}$. ${ }^{\star *} P<0.01$ vs control.

contrast, simulated microgravity for 8,12 , or $24 \mathrm{~h}$ could cause significant ROS production and decrease in locomotion behavior (Fig. 1; Table S1).

Effect of simulated microgravity on expressions of intestinal IncRNAs. In nematodes, some lncRNAs can be expressed in the intestine ${ }^{28-31}$. We further determined the effect of simulated microgravity for $8 \mathrm{~h}$ and $24 \mathrm{~h}$ on expressions of these intestinal lncRNAs in wild-type nematodes. Among these intestinal lncRNAs, simulated microgravity for 8 or $24 \mathrm{~h}$ significantly increased expressions of linc-2, linc-46, linc-61, and linc-78, and decreased expressions of linc-13, linc-14, linc-50, and linc-125 (Fig. 2; Table S2). Moreover, simulated microgravity for $24 \mathrm{~h}$ caused more significant increase in expressions of linc-2, linc-46, linc-61, and linc-78 and decrease in expressions of linc-13, linc-14, linc-50, and linc-125 compared with those in nematodes treated with simulated microgravity for $8 \mathrm{~h}$ (Fig. 2; Table S2).

Effect of RNAi knockdown of linc-2, linc-13, linc-14, linc-46, linc-50, linc-61, linc-78, or linc-125 on toxicity of simulated microgravity. Using ROS production and locomotion behavior as the endpoints, we next examined the effect of RNAi knockdown of linc-2, linc-13, linc-14, linc-46, linc-50, linc-61, linc78 , or linc-125 on toxicity of simulated microgravity in wild-type nematodes. Under the normal conditions, RNAi knockdown of linc-2, linc-13, linc-14, linc-46, linc-50, linc-61, linc-78, or linc-125 did not induce the obvious ROS production and affect the locomotion behavior (Fig. S1; Table S3). After simulated microgravity treatment, RNAi knockdown of linc-46, linc-78, or linc-125 did not noticeably influence the toxicity of simulated microgravity in inducing ROS production and in decreasing locomotion behavior (Fig. S1; Table S3). Different from this, after simulated microgravity treatment, RNAi knockdown of linc-2 or linc-61 led to the more severe toxicity in inducing ROS production and in decreasing locomotion behavior compared with those in wild-type nematodes (Fig. S1; Table S3), suggesting the susceptibility to toxicity of simulated microgravity in linc-2(RNAi) and linc-61(RNAi) nematodes. Additionally, although simulated microgravity for $24 \mathrm{~h}$ did not affect brood size in wild-type nematodes, we observed the significant reduction in brood size in linc-2(RNAi) and linc-61(RNAi) nematodes (Fig. S2; Table S4). In contrast, RNAi knockdown of linc-13, linc-14, or linc-50 suppressed the toxicity of simulated microgravity (Fig. S1; Table S3), suggesting the resistance to toxicity of simulated microgravity in linc-13(RNAi), linc-14(RNAi), and linc-50(RNAi) nematodes. Data on efficiency for RNAi knockdown of linc2, linc-13, linc-14, linc-46, linc-50, linc-61, linc-78, or linc-125 in wild-type nematodes is shown in Fig. S3 and Table S5. 


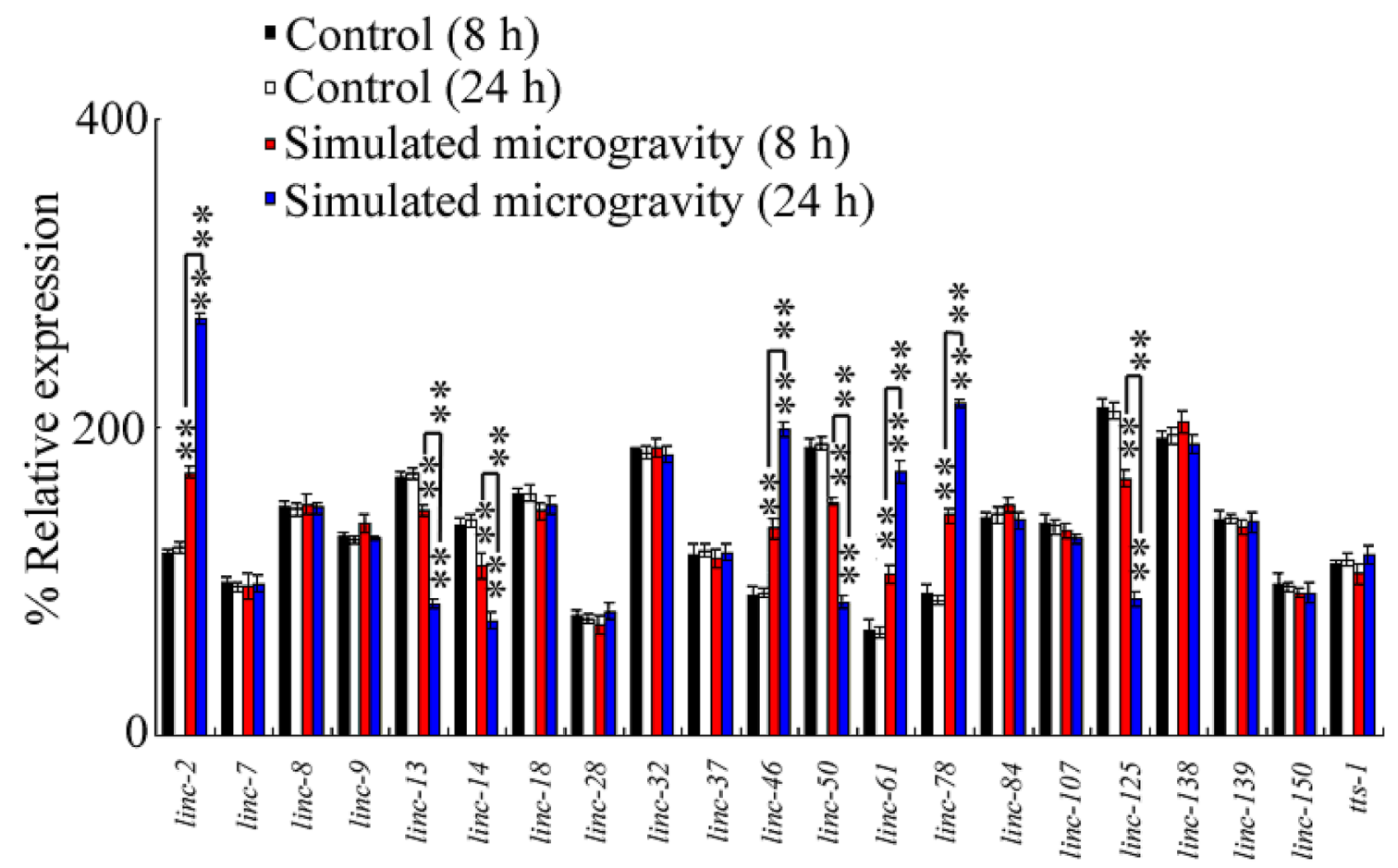

Figure 2. Effect of simulated microgravity on expressions of intestinal lncRNAs in wild-type nematodes. $\mathrm{N}=3$. Simulated microgravity treatment was performed in RCCS system at $30 \mathrm{rpm}$ for 8 or $24 \mathrm{~h}$. Bars represent means \pm SD. ${ }^{* \star} P<0.01$ control ( 8 or $24 \mathrm{~h}$ ) (if not specially indicated).

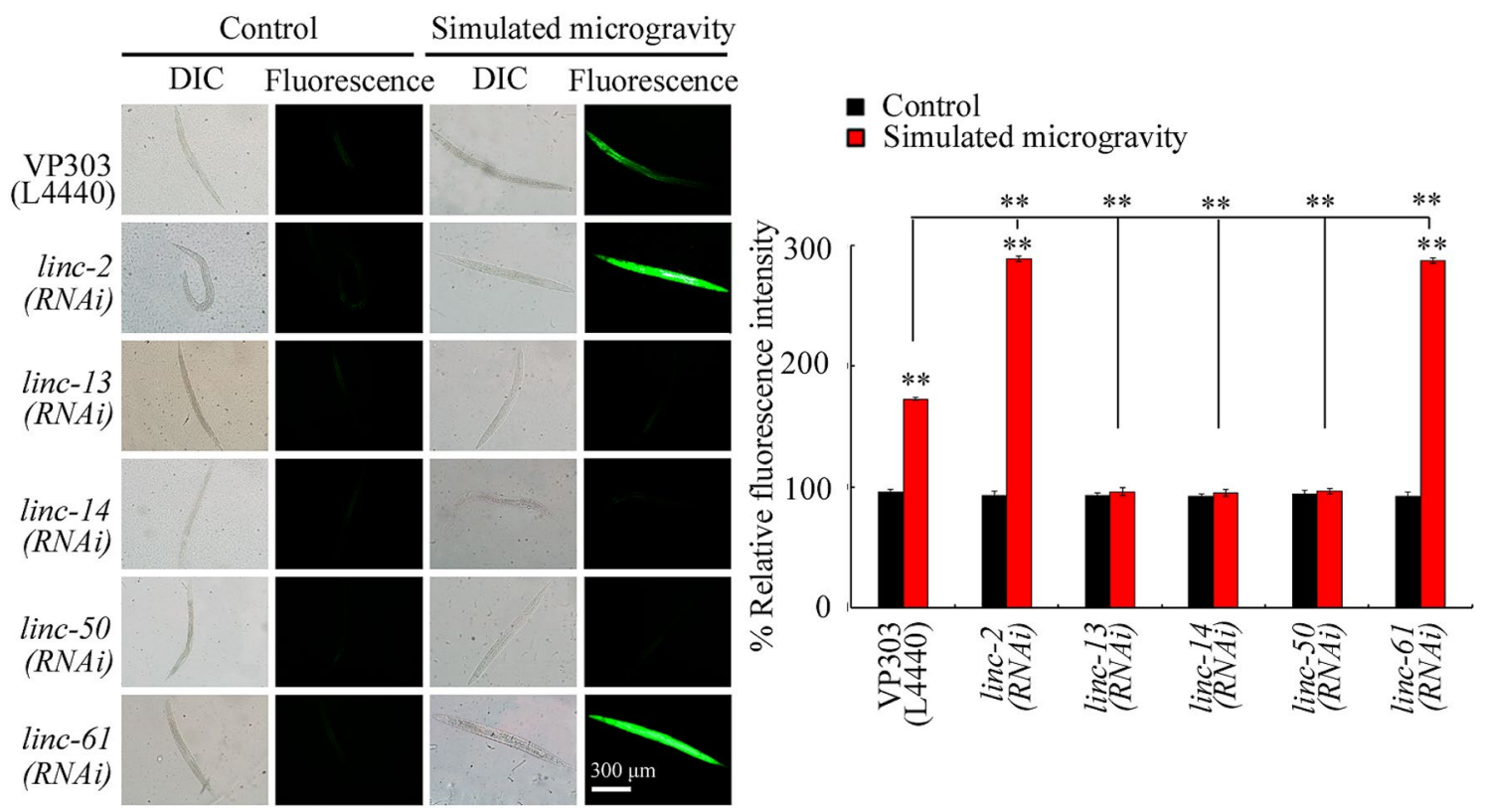

Figure 3. Effect of intestine-specific RNAi knockdown of linc-2, linc-13, linc-14, linc-50, or linc-61 on ROS production in simulated microgravity treated nematodes. $\mathrm{N}=50$. L4440, empty vector. Simulated microgravity treatment was performed in RCCS system at $30 \mathrm{rpm}$ for $24 \mathrm{~h}$. Bars represent means \pm SD. ${ }^{\star \star} P<0.01 v s$ control (if not specially indicated).

Intestine-specific RNAi knockdown of linc-2, linc-13, linc-14, linc-50, or linc-61 affected the toxicity of simulated microgravity. We used VP303 strain to perform RNAi knockdown of certain lncRNA in the intestine ${ }^{32}$. ROS production was used as the endpoint, since the severe defect of locomotion is formed in VP303 ${ }^{32}$. Intestinal RNAi knockdown of linc-2 or linc-61 enhanced the ROS production in simulated microgravity treated VP303 nematodes (Fig. 3; Table S6), suggesting the susceptibility to toxicity of simulated microgravity in animals with intestinal RNAi knockdown of linc-2 or linc-61. However, intestinal RNAi knock- 
a
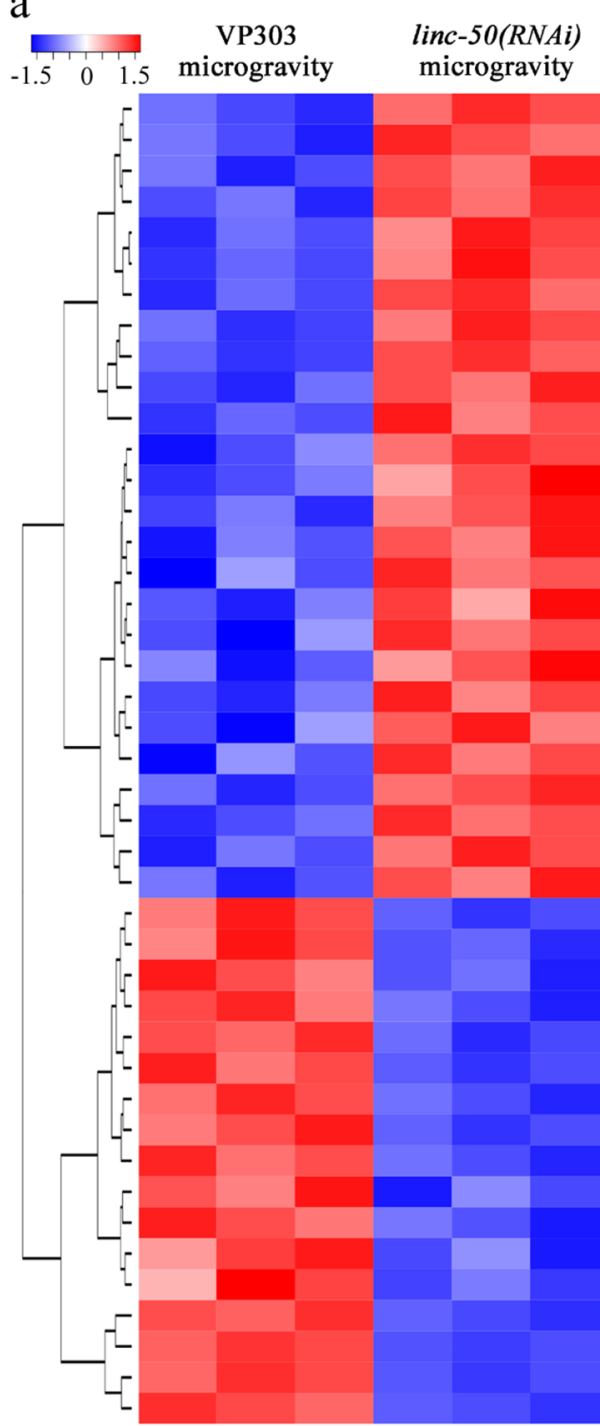

b

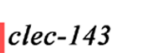

ptr-22

C17B7.5

oac-43

ZK993.5

lbp-7

M04C 9.2

R09H10.2

F42A8.1

T19H5.6

Y73B6BL. 37

skn-1

F54B11.11

daf-16

hlh-30

fipr-1

sodh-2

C25F9.9

C49A1.5

clec-139

C33C12.11

F31F7.1

F15E6.3

B0454. 8

T23E7.6

F49C12.10

Y82E9BL. $3 \mathrm{C}$

aqp-1

R11A5. 3

Y94H6A.10

oac-14

nhr-17

ugt-18

C07G1.7

Y53G8B.2

$f b x b-106$

clec-184

RIIF4.2

fbxa-66

clec-223

F19B2.5

F44E5. 4

C52D10.3

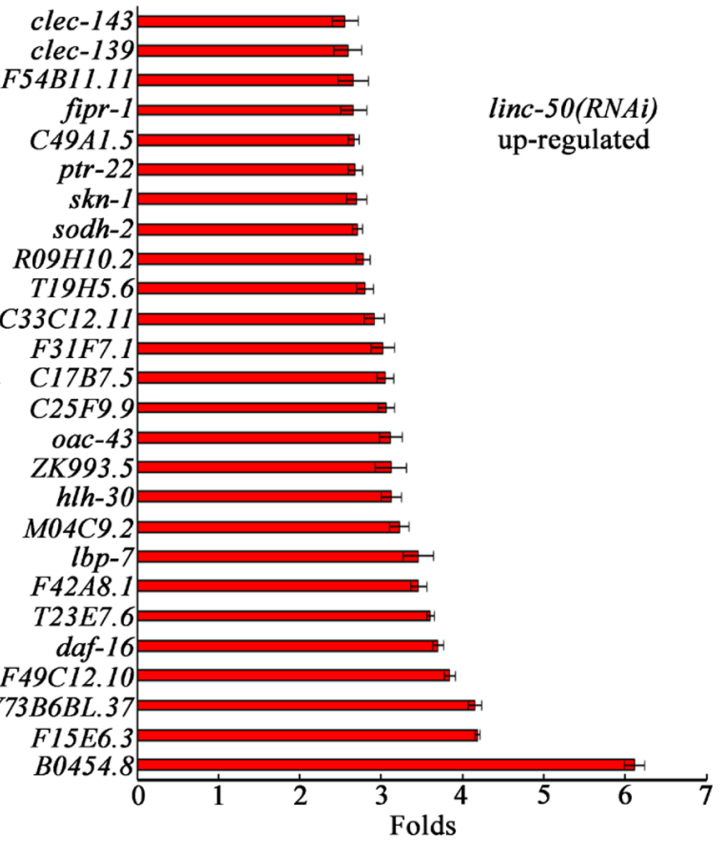

c
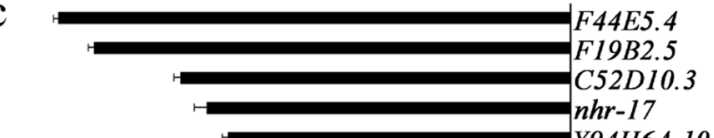

$Y 94 H 6 A .10$
oac-14

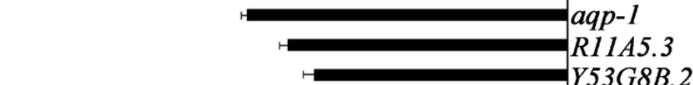

Y53G8B.2

fbxb-106

linc-50(RNAi)

down-regulated fbxa-66

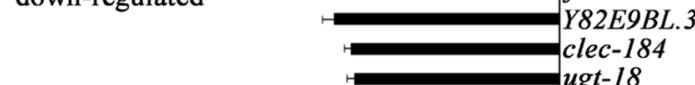

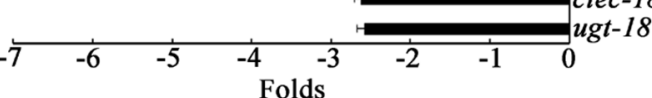

Figure 4. Identification of dysregulated genes by intestine-specific RNAi knockdown of linc-50 in simulated microgravity treated nematodes. (a) Heatmap showing expression of dysregulated genes in simulated microgravity treated nematodes with intestine-specific RNAi knockdown of linc-50. The heatmap was drawn using Omicshare (www.omicshare.com/tools/), a free access online analysis tool of bioinformatics. (b) Upregulated genes induced by intestine-specific RNAi knockdown of linc-50 in simulated microgravity treated nematodes. $\mathrm{N}=3$. (c) Downregulated genes induced by intestine-specific RNAi knockdown of linc-50 in simulated microgravity treated nematodes. $\mathrm{N}=3$. Simulated microgravity treatment was performed in RCCS system at $30 \mathrm{rpm}$ for $24 \mathrm{~h}$.

down of linc-13, linc-14, or linc-50 suppressed the ROS production in simulated microgravity treated VP303 nematodes (Fig. 3; Table S6), suggesting the resistance to toxicity of simulated microgravity in animals with intestinal RNAi knockdown of linc-13, linc-14, or linc-50. Data on efficiency for RNAi knockdown of linc-2, linc13, linc-14, linc-50, or linc-61 in the intestine is shown in Fig. S3 and Table S5.

Identification of dysregulated genes induced by intestine-specific RNAi knockdown of linc-50 in simulated microgravity treated nematodes. We next focused on the identification of downstream genes of linc-50, one of the candidate lncRNAs whose RNAi knockdown induced a resistance to toxicity of simulated microgravity. Based on HiSeq 2000 sequencing, 43 genes were dysregulated by intestine-specific RNAi knockdown of linc-50 in nematodes treated with simulated microgravity for $24 \mathrm{~h}$ (Fig. 4a; Table S7). Meanwhile, expressions of these 43 genes were also affected by simulated microgravity treatment (Table S8). Among these 43 genes, after simulated microgravity treatment, 26 genes (clec-143, clec-139, F54B11.11, fipr-1, C49A1.5, ptr-22, skn-1, sodh-2, R09H10.2, T19H5.6, C33C12.11, F31F7.1, C17B7.5, C25F9.9, oac-43, ZK993.5, hlh-30, M04C9.2, lbp-7, F42A8.1, T23E7.6, daf-16, F49C12.10, Y73B6BL.37, F15E6.3, and B0454.8) were upregulated, and 17 

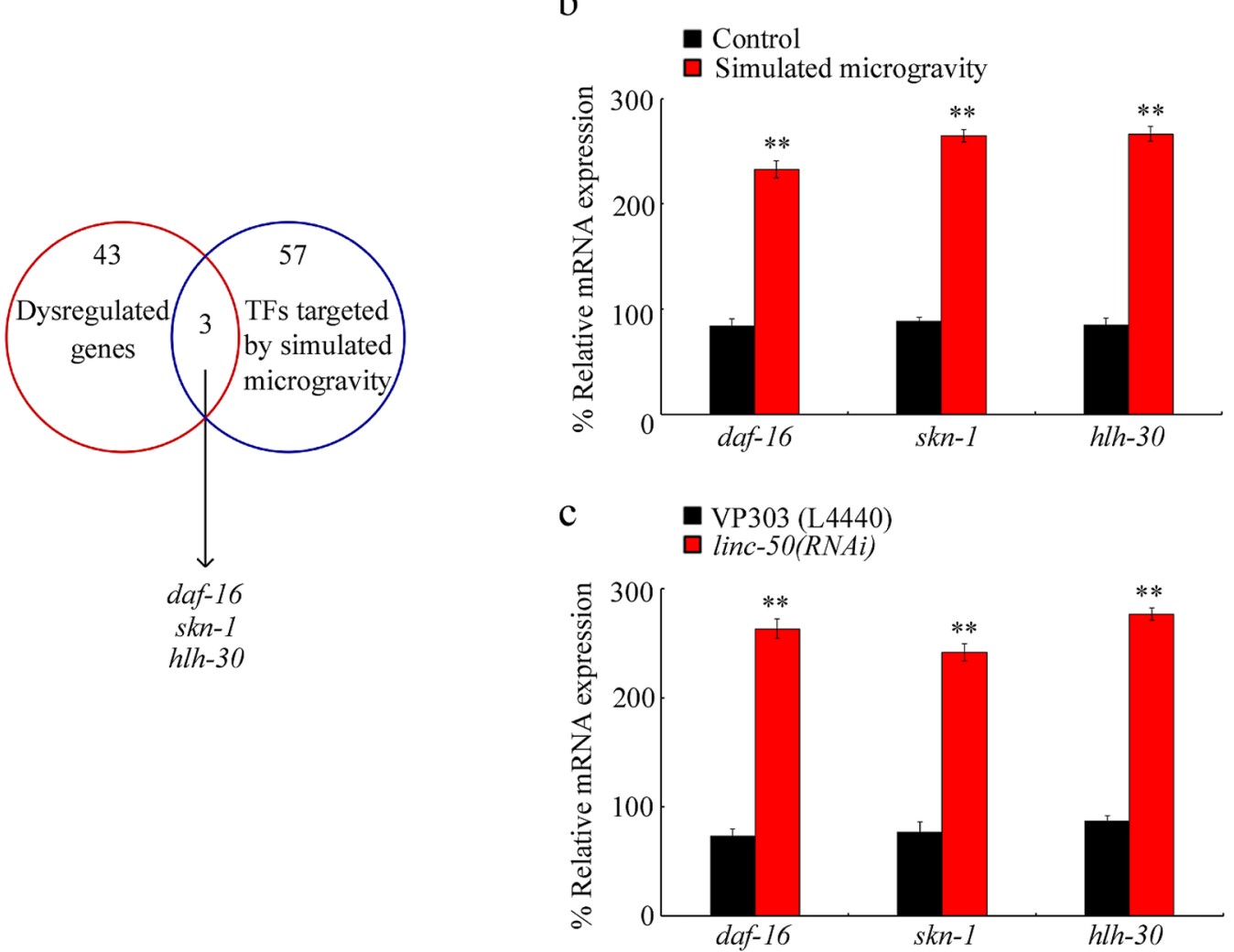

Figure 5. Screen of possible downstream transcriptional factors targeted by intestinal linc-50 in simulated microgravity treated nematodes. (a) DAF-16, SKN-1, and HLH-30 are candidate transcriptional factors targeted by linc-50 in simulated microgravity treated nematodes. TFs, transcriptional factors. (b) Effect of simulated microgravity on expressions of $d a f-16, s k n-1$, and $h l h-30 . \mathrm{N}=3$. Bars represent means $\pm \mathrm{SD} .{ }^{\star *} P<0.01 v s$ control. (c) Effect of intestine-specific RNAi knockdown of linc-50 on expressions of daf-16, skn-1, and hlh-30 in simulated microgravity treated nematodes. $\mathrm{N}=3$. L4440, empty vector. Bars represent means $\pm \mathrm{SD}$. ${ }^{\star *} P<0.01 v s$ VP303. Simulated microgravity treatment was performed in RCCS system at $30 \mathrm{rpm}$ for $24 \mathrm{~h}$.

genes (F44E5.4, F19B2.5, C52D10.3, nhr-17, Y94H6A.10, oac-14, aqp-1, R11A5.3, Y53G8B.2, R11F4.2, fbxa-106, C07G1.7, clec-223, fbxa-66, Y82E9BL.3, clec-184, and ugt-18) were downregulated (Fig. 4b,c; Table S9).

Potential transcriptional factors targeted by intestinal linc-50 in simulated microgravity treated nematodes. During the control of biological processes, lncRNAs can act as 'ligands' of transcriptional factors ${ }^{33}$. In nematodes, the candidate transcriptional factors targeted by lncRNAs have been identified by ChIP-SEQ technique to determine transcriptional factors binding site regions of lncRNAs, together with antibodies against transcriptional factors and immunoprecipitate nucleic acids ${ }^{34,35}$. Among the dysregulated genes induced by simulated microgravity treatment, 3 genes (daf-16, skn-1, and hlh-30) encode transcriptional factors potentially targeted by linc-50 (Fig. 5a). The treatment with simulated microgravity could significantly increase expressions of $d a f-16, s k n-1$, and $h l h-30$ (Fig. 5b; Table S10). Simulated microgravity treatment further increased both expression and nucleus localization of DAF-16::GFP in intestinal cells (Fig. S4a; Table S11). Similarly, the simulated microgravity also increased both expression and nucleus localization of SKN-1::GFP in intestinal cells (Fig. S4b; Table S11). Moreover, in simulated microgravity treated nematodes, intestine-specific RNAi knockdown of linc-50 significantly increased expressions of daf-16, skn-1, and hlh-30 (Fig. 5c; Table S10). Additionally, after simulated microgravity treatment, both expression and nucleus localization of DAF-16::GFP were increased by RNAi knockdown of linc-50 (Fig. S4; Table S11). These data implied that DAF-16, SKN-1, and HLH-30 might function as the targeted transcriptional factors by intestinal linc-50 in simulated microgravity treated nematodes. DAF-16 is a FOXO transcriptional factor in insulin signaling pathway involved in the regulation of response to simulated microgravity ${ }^{21}$. SKN-1 is a Nrf protein in p38 MAPK signaling pathway, and was required for the regulation of response to simulated microgravity ${ }^{23}$. HLH-30 is a transcriptional factor governing the autophagy induction, and required for the regulation of stress response $\mathrm{e}^{22}$.

PRIdictor (protein-RNA interaction predictor) is a tool to predict mutual binding sites in RNA and protein at nucleotide or residue level (http://bclab.inha.ac.kr/pridictor). PRIdictor was further used to predict binding sites between linc-50 and DAF-16, SKN-1, or HLH-30. The DAF-16 protein contains 25 possible nucleotide binding sites for linc-50 (Fig. S5a), and the linc-50 contains 40 possible residue binding sites for DAF-16 (Fig. S5b). The SKN-1 protein contains 25 possible nucleotide binding sites for linc-50 (Fig. S5c), and the linc-50 contains 82 


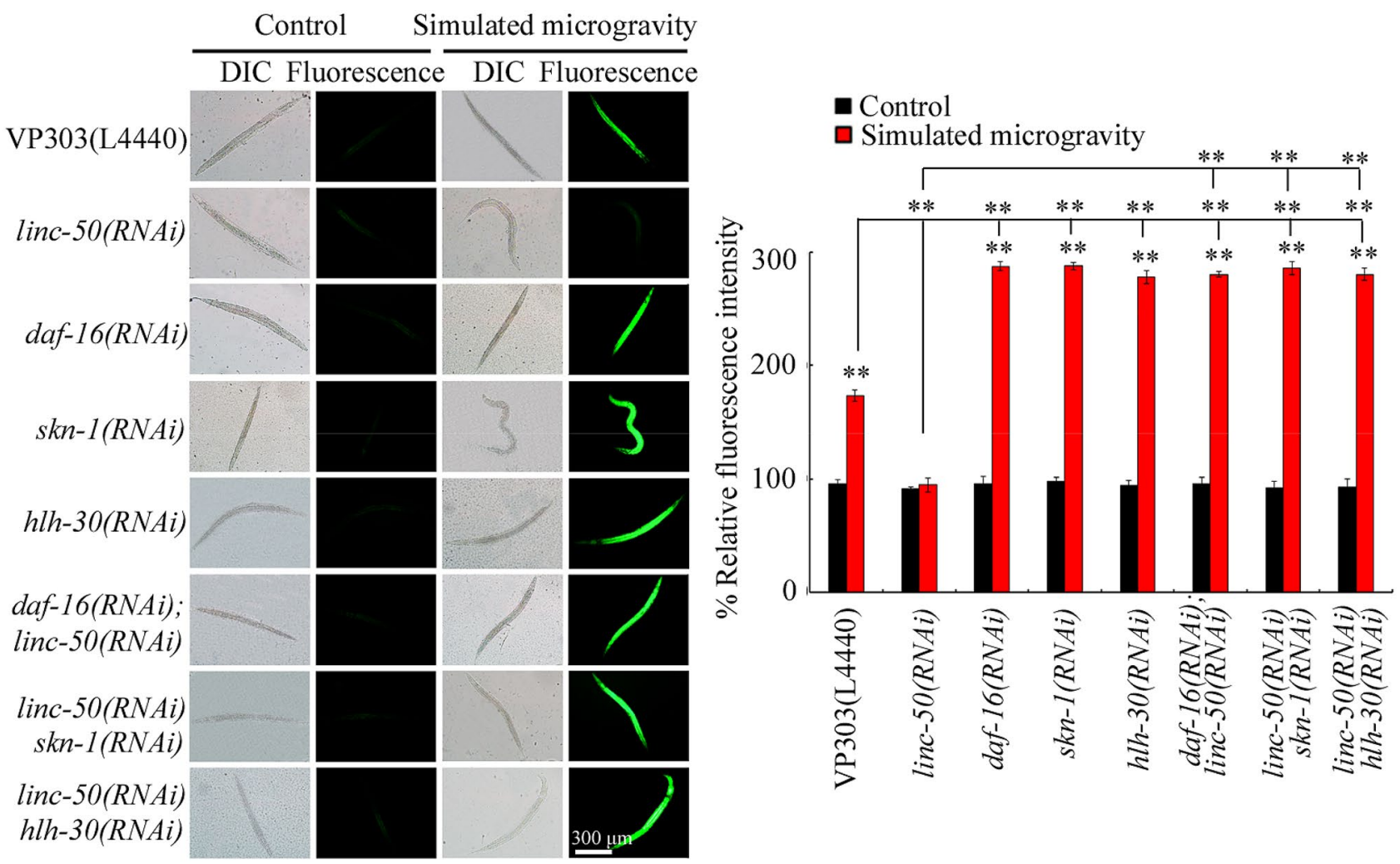

Figure 6. Genetic interaction between linc-50 and DAF-16, SKN-1, or HLH-30 in the intestine to regulate ROS production in simulated microgravity treated nematodes. $\mathrm{N}=50$. L4440, empty vector. Simulated microgravity treatment was performed in RCCS system at $30 \mathrm{rpm}$ for $24 \mathrm{~h}$. Bars represent means \pm SD. ${ }^{* *} P<0.01 v s$ control (if not specially indicated).

possible residue binding sites for SKN-1 (Fig. S5d). The HLH-30 protein contains 2 possible nucleotide binding sites for linc-50 (Fig. S5e), and the linc-50 contains 5 possible residue binding sites for HLH-30 (Fig. S5f).

Genetic interaction between linc-50 and DAF-16, SKN-1, or HLH-30 in the intestine to regulate response to simulated microgravity. RNAi knockdown of daf-16, skn-1, or hlh-30 in the intestine increased ROS production in simulated microgravity treated VP303 nematodes (Fig. 6; Table S12), suggesting the susceptibility to toxicity of simulated microgravity in nematodes with intestinal RNAi knockdown of daf-16, $s k n-1$, or hlh-30. Moreover, intestinal RNAi knockdown of $d a f-16, s k n-1$, or $h l h-30$ inhibited the resistance to toxicity of simulated microgravity in linc-50(RNAi) nematodes (Fig. 6; Table S12). Therefore, in the intestine, DAF-16, SKN-1, and HLH-30 functioned downstream of linc-50 to control response to simulated microgravity. Data on efficiency for intestinal RNAi knockdown of linc-50, daf-16, skn-1, or hlh-30 is shown in Figs. S6, S7 and Tables S13, S14.

Genetic interaction among DAF-16, SKN-1, and HLH-30 in the intestine to regulate response to simulated microgravity. After simulated microgravity treatment, genetic interaction analysis indicated that double intestine-specific RNAi knockdown of $d a f-16$ and $s k n-1$ could cause the more severe ROS production than that in daf-16(RNAi) or skn-1(RNAi) nematodes (Fig. 7a; Table S15), suggesting that DAF-16 and SKN-1 acted in parallel pathways to regulate response to simulated microgravity. Similarly, after simulated microgravity treatment, double intestine-specific RNAi knockdown of daf-16 and hlh-30 could cause the more severe ROS production than that in daf-16(RNAi) or hlh-30(RNAi) nematodes (Fig. 7a; Table S15), suggesting that DAF-16 and HLH-30 also acted in parallel pathways to regulate response to simulated microgravity. Moreover, double intestine-specific RNAi knockdown of $s k n-1$ and $h l h$-30 led to the more severe ROS production in simulated microgravity treated nematodes than that in simulated microgravity treated skn-1(RNAi) or hlh-30(RNAi) nematodes (Fig. 7a; Table S15), suggesting that SKN-1 and HLH-30 further acted in parallel pathways to regulate response to simulated microgravity. Data on efficiency for intestinal RNAi knockdown of daf-16, skn-1, or hlh-30 is shown in Figs. S6, S7 and Tables S13, S14.

\section{Discussion}

Our previous studies have implied the crucial role of intestinal barrier in response to simulated microgravity in nematodes ${ }^{19,21,23-25}$. Firstly, simulated microgravity could activate the protective responses (such as mt UPR) in intestinal cells ${ }^{21}$. Secondly, simulated microgravity could cause damage on intestinal barrier, such as the enhanced intestinal permeability ${ }^{19}$. Thirdly, some important signaling pathways (such as insulin, p38 MAPK, 
A

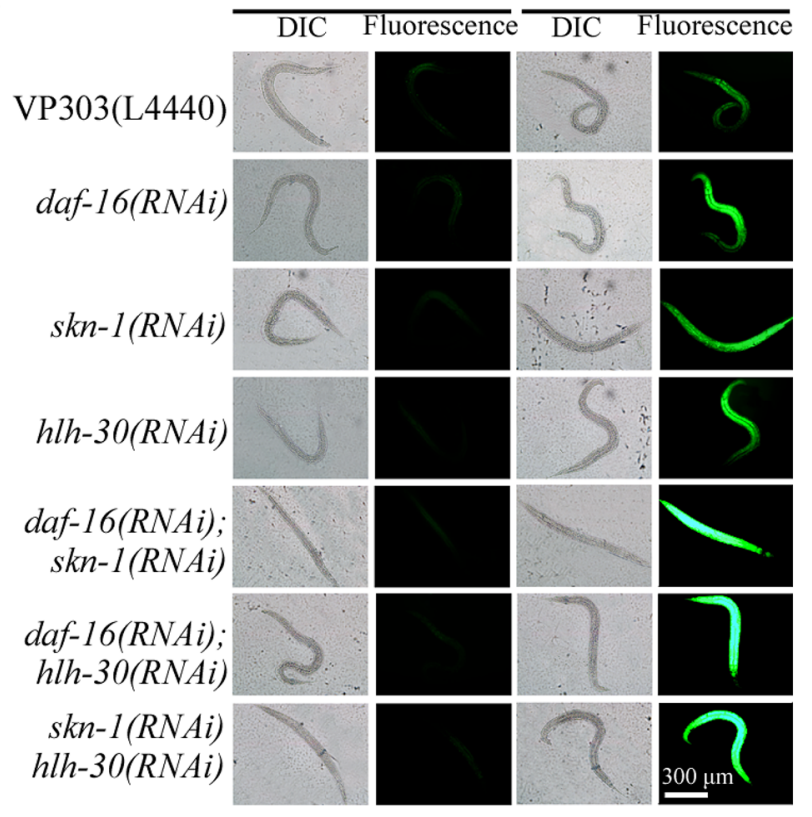

Control

Simulated microgravity

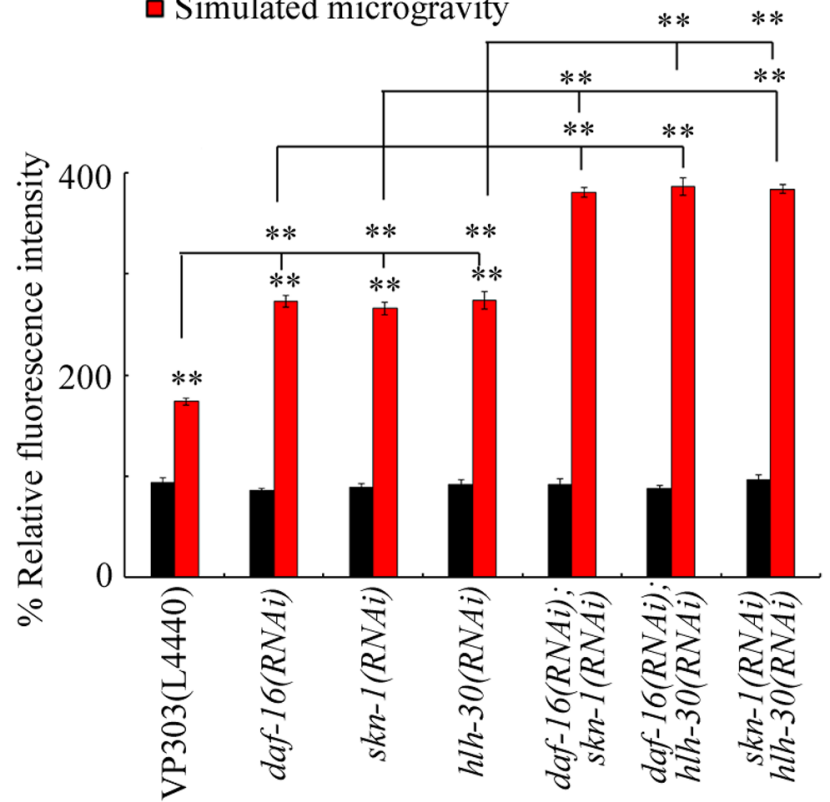

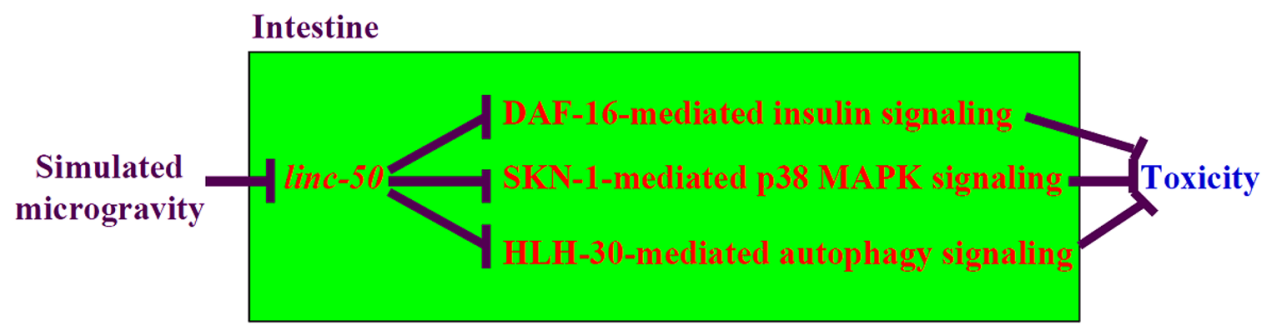

Figure 7. Genetic interaction among DAF-16, SKN-1, and HLH-30 in the intestine to regulate the response to simulated microgravity in nematodes. (a) Genetic interaction among DAF-16, SKN-1, and HLH-30 in the intestine to regulate the toxicity of simulated microgravity in inducing ROS production. $\mathrm{N}=50$. L4440, empty vector. Simulated microgravity treatment was performed in RCCS system at $30 \mathrm{rpm}$ for $24 \mathrm{~h}$. Bars represent means \pm SD. ${ }^{* *} P<0.01 v s$ control (if not specially indicated). (b) A diagram showing the molecular basis of intestinal linc-50 in regulating the response to simulated microgravity in nematodes.

and Wnt signaling pathways) could be activated in the intestine, and were involved in the regulation of response to simulated microgravity ${ }^{23-25}$. Thus, in this study, we tried to identify the intestinal lncRNAs involved in the regulation of response to simulated microgravity in nematodes.

Among intestinal lncRNAs, simulated microgravity treatment for 8 or $24 \mathrm{~h}$ could increase expressions of linc2, linc-46, linc-61, and linc-78, and decrease expressions of linc-13, linc-14, linc-50, and linc-125 (Fig. 2; Table S2). Previous study has also suggested that exposure to $1 \mathrm{mg} / \mathrm{L}$ graphene oxide (GO) could decrease expression of linc- $14^{28}$. Additionally, exposure to $1-100 \mu \mathrm{g} / \mathrm{L}$ nanopolystyrene $(100 \mathrm{~nm})$ could also increase expressions of linc-2 and linc-61, and decrease linc-50 expression ${ }^{29}$. These results implied the possible conserved property of linc-2, linc-14, linc-50, and linc-61 in response to environmental toxicants or stresses.

Among the 8 dysregulated intestinal lncRNAs in simulated microgravity treated nematodes, we further observed the susceptibility of linc-2(RNAi) and linc-61(RNAi) nematodes to toxicity of simulated microgravity and the resistance of linc-13(RNAi), linc-14(RNAi), and linc-50(RNAi) nematodes to toxicity of simulated microgravity (Fig. S1; Table S3). These observations suggested that the alteration in expressions of linc-2, linc-13, linc-14, linc-50, and linc-61 mediated a protective response to simulated microgravity. The resistance to toxicity of GO could also be detected in linc-14(RNAi) nematodes ${ }^{28}$. Additionally, RNAi knockdown of linc-2 or linc-61 could result in the susceptibility to toxicity of nanopolystyrene, and RNAi knockdown of linc-50 caused the resistance to toxicity of nanopolystyrene ${ }^{29}$.

Moreover, our results demonstrated the important functions of linc-2, linc-13, linc-14, linc-50, and linc-61 in the intestine to regulate response to simulated microgravity. Nevertheless, besides the expression in the intestine, linc-2, linc-13, linc-50, and linc-61 can also be expressed in other tissues in nematodes ${ }^{31}$. Thus, we did not exclude the possibility that these 5 candidate lncRNAs can also act in other tissues to regulate the response to simulated microgravity.

For the linc-50, we found that it functioned upstream of three transcriptional factors (DAF-16, SKN-1, and HLH-30) to control response to simulated microgravity (Fig. 5a,b, Fig. S5; Table S10). In nematodes, DAF-16, SKN-1, and HLH-30 could act in the intestine to regulate response to simulated microgravity (Fig. 6; 
Table S12 $)^{23,24}$. Therefore, linc-50-DAF-16, linc-50-SKN-1, and linc-50-HLH-30 could be formed in the intestine to regulate response to simulated microgravity.

Moreover, our data suggested that intestinal linc-50 regulated the response to simulated microgravity by targeting and suppressing transcriptional factors of DAF-16, SKN-1, and HLH-30 (Figs. 5c, 6; Tables S10, S12). Our results have indicated the important role of linc-50 in response to both environmental stress and environmental toxicants ${ }^{29}$. These observations provided an important molecular basis for our understanding the role of linc-50 in response to environmental stress or toxicants. Under the normal conditions, intestinal RNAi knockdown of linc-50 could increase expressions of $d a f-16$ and $h l h-30$, but did not affect $s k n-1$ expression (Fig. S8; Table S16).

It was further found that DAF-16, SKN-1, and HLH-30 functioned synergistically to regulate response to simulated microgravity (Fig. 7a; Table S15). Therefore, intestinal linc-50 regulated response to simulated microgravity by simultaneously suppressing functions of downstream DAF-16-medaied insulin signaling, SKN-1-mediated p38 MAPK signaling, and HLH-30-medaited autophagy signaling (Fig. 7b; Table S15). Insulin signaling, p38 MAPK signaling, and autophagy signaling reflect three forms of protective responses to environmental toxicants or stresses ${ }^{7,22}$, which further indicated the crucial role of intestinal linc-50 in regulating the response to simulated microgravity.

In conclusion, we used C. elegans as an animal model to determine the functions of intestinal lncRNAs in regulating response to simulated microgravity stress. We identified 5 intestinal lincRNAs with protective functions during the control of response to simulated microgravity. Moreover, we found that intestinal linc-50 could regulate response to simulated microgravity by suppressing functions of downstream three transcriptional factors (DAF-16, SKN-1, and HLH-30). Our study provides an important molecular basis for lncRNAs in the intestine to regulate response to environmental exposures in nematodes.

\section{Methods}

Strains and maintenance. The used wild-type nematodes are Bristol N2. Wild-type, zIs356[Pdaf-16::daf16a/b::GFP], ldEx9[skn-1::GFP], and VP303/rde-1(ne219);kbIs7 strains were from Caenorhabditis Genetics Center (CGC). Nematodes were normally maintained on normal nematode growth mediate (NGM) plates fed with Escherichia coli OP50 as a food source ${ }^{36}$. To obtain synchronized L1-larvae or young adults, the gravid nematodes were treated with bleaching solution $(5 \% \mathrm{NaOCl} / 1 \mathrm{~N} \mathrm{NaOH}, 2: 5)$ to release enough eggs. After that, the eggs were transferred onto another plate to allow the further development into L1-larvae or young adult population.

Simulated microgravity. Simulated microgravity was carried out as described ${ }^{19}$. In cultivation chamber of the Rotary System (Synthecon), the vessels containing soft and movable $0.2 \%$ agar medium with suspended young adults were half filled. In vessels containing soft and movable $0.2 \%$ agar medium, no E. coli OP50 was added. The simulated microgravity was generated after balancing sedimentation-induced gravity with centrifugation by Rotary Cell Culture System (RCCS) vessel rotation. To prepare this balancing state, different vessels were placed symmetrically on RCCS system. To set up simulated microgravity, RCCS rotated chamber horizontally at $30 \mathrm{rpm}$ for $4,8,12$, or $24 \mathrm{~h}$ at $20^{\circ} \mathrm{C}$. Control young adults were grown in soft and movable $0.2 \%$ agar medium without simulated microgravity treatment at $20^{\circ} \mathrm{C}$.

Quantitative real-time polymerase chain reaction (qRT-PCR). After simulated microgravity treatment, total RNAs were isolated using Trizol (Sigma-Aldrich). Synthesis of cDNAs using reverse transcriptase reaction was prepared in Mastercycler gradient PCR system (Eppendorf). Transcriptional expressions of examined lncRNAs (linc-2, linc-7, linc-8, linc-9, linc-13, linc-14, linc-18, linc-28, linc-32, linc-37, linc-46, linc-50, linc61, linc-78, linc-107, linc-125, linc-138, linc-139, linc-150, and tts-1) and genes (daf-16, skn-1, and hlh-30) were determined using SYBR Green qRT-PCR master mix (TOYOBO, Japan) in StepOnePlus real-time PCR system (Applied Biosystems). tba-1 encoding an alpha-tubulin protein was used as a reference gene. Three replicates were carried out for all the reactions. Primer information for qRT-PCR T is shown in Table S17.

RNA interference (RNAi). Escherichia coli $\mathrm{HT} 115$ was cultured in LA broth (LB broth containing $100 \mu \mathrm{g} / \mathrm{L}$ ampicillin) with the addition of $5 \mathrm{mM}$ isopropyl 1-thio- $\beta$-D-galactopyranoside (IPTG). L1-larvae were maintained on NGM plates fed with E. coli HT115 for RNAi knockdown of linc-2, linc-13, linc-14, linc-46, linc-50, linc-61, linc-78, linc-125, daf-16, skn-1, or $h l h-30^{37}$. Once the nematodes developed into the gravid, they were transferred onto new RNAi plates. The second generation was used for simulated microgravity treatment. HT115 expressing empty vector L4440 was used as a control. Transgenic strain of VP303 is a genetic tool for intestinal RNAi knockdown of gene $(s)^{32}$. qRT-PCR was performed to confirm RNAi knockdown efficiency.

Toxicity assessment. Considering the sensitivity in assessing toxicity of simulated microgravity ${ }^{18,23,24}$, locomotion behavior and ROS production were used as toxicity assessment endpoints.

Locomotion behavior reflects functional state of motor neurons, and was evaluated by head thrash and body bend ${ }^{38}$. After the treatment, nematodes were washed using M9 buffer first. The nematodes were allowed for a 1 -min recovery on surface of NMG plate. Under a dissecting microscopy, a head thrash is defined as an alteration in direction of posterior bulb part, and the head thrash frequency was recorded during the duration of $1 \mathrm{~min}$. A body bend is defined as an alteration in direction of bending at the middle body, and the body bend frequency was recorded during the duration of $20 \mathrm{~s}$. Forty nematodes were analyzed per treatment. Three replicates were performed.

ROS production was used to assess activation of oxidative stress in simulated microgravity treated nematodes $^{39,40}$. After the treatment, nematodes were labeled with $1 \mu \mathrm{M} C \mathrm{M}-\mathrm{H}_{2} \mathrm{DCFDA}$ for 3-h without light. 
After that, the nematodes were examined at $510 \mathrm{~nm}$ of emission filter and at $488 \mathrm{~nm}$ of excitation wavelength under a laser scanning confocal microscope (Leica, TCS SP2, Bensheim, Germany). Before the observations, the nematodes were paralyzed by adding sodium azide $(0.01 \mathrm{M})$ on the agar pad. The $20 \times$ objective was used for the observations. In nematodes, the strongest ROS fluorescent signals can be detected in the intestine ${ }^{41}$. Semiquantification was determined for fluorescence intensity in the intestine in comparison to autofluorescence. Fifty animals were analyzed per treatment. Three replicates were performed.

Brood size was selected as an endpoint to reflect reproductive capacity ${ }^{42}$. To determine the brood size, number of offspring at all stages beyond the eggs was counted under a stereomicroscopy. For each treatment, thirty nematodes were examined. Three replicates were performed.

HiSeq 2000 sequencing. HiSeq 2000 sequencing was used to identify dysregulated genes induced by intestinal RNAi knockdown of linc-50 in simulated microgravity treated nematodes. The mRNA libraries were prepared with RNA-seq Sample Preparation kit (Illumina, Inc., USA) for Illumina HiSeq 2000 sequencing. Fast QC was used to check the quality of reads. Total read numbers of data sets were normalized to equal levels. The dysregulated genes were judged by statistical significance analysis and use of a 2.0 -fold change cutoff.

Statistical analysis. SPSS 12.0 was used for statistical analysis. One-way analysis of variance (ANOVA) was used to determine differences between groups. Two-way ANOVA analysis was used for multiple factor comparisons. Differences within groups were determined using Dunnett- $t$ test, and probability level 0.01 was considered as statistically significant.

Received: 17 July 2020; Accepted: 4 January 2021

Published online: 21 January 2021

\section{References}

1. Altman, P. L. \& Talbot, J. M. Nutrition and metabolism in spaceflight. J. Nutr. 117, 421-427 (1987).

2. Longnecker, D. E., Manning, F. J. \& Worth, M. H. Jr. Review of NASA's Longitudinal Study of Astronaut Health 94 (The National Academic Press, Washington, 2004).

3. Fitts, R. H. et al. Prolonged space flight-induced alterations in the structure and function of human skeletal muscle fibres. J. Physiol. 588, 3567-3592 (2010)

4. Steinberg, F., Kalicinski, M., Dalecki, M. \& Bock, O. Human performance in a realistic instrument-control task during short-term microgravity. PLoS One 10, e0128992 (2015).

5. Seibert, F. S. et al. The effect of microgravity on central aortic blood pressure. Am. J. Hypertension 31, 1183-1189 (2018).

6. Wang, D.-Y. Nanotoxicology in Caenorhabditis elegans (Springer, Berlin, 2018).

7. Wang, D.-Y. Target Organ Toxicology in Caenorhabditis elegans (Springer, Berlin, 2019).

8. Qiu, Y.-X., Liu, Y.-Q., Li, Y.-H., Li, G.-J. \& Wang, D.-Y. Effect of chronic exposure to nanopolystyrene on nematode Caenorhabditis elegans. Chemosphere 256, 127172 (2020).

9. Yang, Y.-H., Wu, Q.-L. \& Wang, D.-Y. Epigenetic response to nanopolystyrene in germline of nematode Caenorhabditis elegans. Ecotoxicol. Environ. Saf. 206, 111404 (2020).

10. Liu, H.-L. \& Wang, D.-Y. Intestinal mitochondrial unfolded protein responseinduced by nanoplastic particles in Caenorhabditis elegans. Chemosphere. https://doi.org/10.1016/j.chemosphere.2020.128917 (2020).

11. Higashitani, A. et al. Checkpoint and physiological apoptosis in germ cells proceeds normally in spaceflown Caenorhabditis elegans. Apoptosis 10, 949-954 (2005).

12. Szewczyk, N. J. et al. Caenorhabditis elegans survives atmospheric breakup of STS-107, space shuttle Columbia. Astrobiology 5, 690-705 (2005).

13. Higashibata, A. et al. Decreased expression of myogenic transcriptional factors and myosin heavy chains in Caenorhabditis elegans muscles developed during spaceflight. J. Exp. Biol. 209, 3209-3218 (2006).

14. Adenle, A. A., Johnsen, B. \& Szewczyk, N. J. Review of the results from the International C. elegans first experiment (ICE-FIRST). Adv. Sp. Res. 44, 210-216 (2009).

15. Gao, Y., Li, S., Xu, D., Wang, J. \& Sun, Y. Changes in apoptotic microRNA and mRNA expression profiling in Caenorhabditis elegans during the Shenzhou-8 mission. J. Radiat. Res. 56, 782-882 (2015).

16. Zhao, L., Gao, Y., Mi, D. \& Sun, Y. Mining potential biomarkers associated with space flight in Caenorhabditis elegans experienced Shenzhou-8 mission with multiple feature selection techniques. Mutat. Res. 791-792, 27-34 (2016).

17. Higashibata, A. et al. Microgravity elicits reproducible alterations in cytoskeletal and metabolic gene and protein expression in space-flown Caenorhabditis elegans. NPG Micrograv. 2, 15022 (2016).

18. Zhao, L., Rui, Q. \& Wang, D.-Y. Molecular basis for oxidative stress induced by simulated microgravity in nematode Caenorhabditis elegans. Sci. Total Environ. 607-608, 1381-1390 (2017)

19. Liu, H.-L., Guo, D.-Q., Kong, Y., Rui, Q. \& Wang, D.-Y. Damage on functional state of intestinal barrier by microgravity stress in nematode Caenorhabditis elegans. Ecotoxicol. Environ. Saf. 183, 109554 (2019).

20. Sun, L.-L., Li, W.-J., Li, D. \& Wang, D.-Y. microRNAs involved in the control of toxicity on locomotion behavior induced by simulated microgravity stress in Caenorhabditis elegans. Sci. Rep. 10, 17510 (2020).

21. Liu, P.-D., Li, D., Li, W.-J. \& Wang, D.-Y. Mitochondrial unfolded protein response to microgravity stress in nematode Caenorhabditis elegans. Sci. Rep. 9, 16474 (2019).

22. Wang, D.-Y. Molecular Toxicology in Caenorhabditis elegans (Springer, Berlin, 2019).

23. Li, W.-J., Wang, D.-Y. \& Wang, D.-Y. Regulation of the response of Caenorhabditis elegans to simulated microgravity by p38 mitogen-activated protein kinase signaling. Sci. Rep. 8, 857 (2018).

24. Kong, Y., Liu, H.-L., Li, W.-J. \& Wang, D.-Y. Intestine-specific activity of insulin signaling pathway in response to microgravity stress in Caenorhabditis elegans. Biochem. Biophys. Res. Commun. 517, 278-284 (2019).

25. Rui, Q., Dong, S.-S., Jiang, W.-K. \& Wang, D.-Y. Response of canonical Wnt/ $\beta$-catenin signaling pathway in the intestine to microgravity stress in Caenorhabditis elegans. Ecotoxicol. Environ. Saf. 186, 109782 (2019).

26. Quinn, J. J. \& Chang, H. Y. Unique features of long non-coding RNA biogenesis and function. Nat. Rev. Genet. 17, 47-62 (2016).

27. Ransohoff, J., Wei, Y. \& Khavari, P. A. The functions and unique features of long intergenic non-coding RNA. Nat. Rev. Mol. Cell Biol. 19, 143-157 (2018). 
28. Wu, Q.-L. et al. Genome-wide identification and functional analysis of long noncoding RNAs involved in the response to graphene oxide. Biomaterials 102, 277-291 (2016).

29. Qu, M. et al. Identification of long non-coding RNAs in response to nanopolystyrene in Caenorhabditis elegans after long-term and low-dose exposure. Environ. Pollut. 255, 113137 (2019).

30. Essers, P. B. et al. A long noncoding RNA on the ribosome is required for lifespan extension. Cell Rep. 10, 339-345 (2015).

31. Liu, W. et al. Spatiotemporal expression profiling of long intervening noncoding RNAs in Caenorhabditis elegans. Sci. Rep. 7, 5195 (2017).

32. Espelt, M. V., Estevez, A. Y., Yin, X. \& Strange, K. Oscillatory $\mathrm{Ca}^{2+}$ signaling in the isolated Caenorhabditis elegans intestine: Role of the inositol-1,4,5-trisphosphate receptor and phospholipases C beta and gamma. J. Gener. Physiol. 126, 379-392 (2005).

33. Chen, L. L. \& Carmichael, G. G. Decoding the function of nuclear long non-coding RNAs. Curr. Opin. Cell Biol. 22, 357-364 (2010).

34. Zhong, M. et al. Genome-wide identification of binding sites defines distinct functions for Caenorhabditis elegans PHA-4/FOXA in development and environmental response. PLoS Genet. 6, e1000848 (2010).

35. Niu, W. et al. Diverse transcription factor binding features revealed by genome-wide ChIP-seq in C. elegans. Genome Res. 21, 245-254 (2011).

36. Brenner, S. The genetics of Caenorhabditis elegans. Genetics 77, 71-94 (1974).

37. Li, D., Yuan, Y.-J. \& Wang, D.-Y. Regulation of response to nanopolystyrene by intestinal microRNA mir-35 in nematode Caenorhabditis elegans. Sci. Total Environ. 736, 139677 (2020).

38. Liu, H.-L., Zhang, R.-J. \& Wang, D.-Y. Response of DBL-1/TGF- $\beta$ signaling-mediated neuron-intestine communication to nanopolystyrene in nematode Caenorhabditis elegans. Sci. Total Environ. 745, 1141047 (2020).

39. Qiu, Y.-X., Liu, Y.-Q., Li, Y.-H. \& Wang, D.-Y. Intestinal mir-794 responds to nanopolystyrene by linking insulin and p38 MAPK signaling pathways in nematode Caenorhabditis elegans. Ecotoxicol. Environ. Saf. 201, 110857 (2020).

40. Wang, S.-T., Liu, H.-L., Zhao, Y.-Y., Rui, Q. \& Wang, D.-Y. Dysregulated mir-354 enhanced the protective response to nanopolystyrene by affecting the activity of TGF- $\beta$ signaling pathway in nematode Caenorhabditis elegans. NanoImpacts 20, 100256 (2020).

41. Wang, S. et al. Induction of germline cell cycle arrest and apoptosis by sodium arsenite in Caenorhabditis elegans. Chem. Res. Toxicol. 29, 181-186 (2007).

42. Yang, Y.-H., Du, H.-H., Xiao, G.-S., Wu, Q.-L. \& Wang, D.-Y. Response of intestinal Ga subunits to nanopolystyrene in nematode Caenorhabditis elegans. Environ. Sci. Nano 7, 2351-2359 (2020).

\section{Acknowledgements}

This study was supported by the Grant of Fundamental Research Funds for the Central Universities (2242020k1G015).

\section{Author contributions}

Conceived and designed the experiments: D.W. Performed the experiments and analyzed the data: L.S., D.L. and Y.Y. Wrote the paper: D.W.

\section{Competing interests}

The authors declare no competing interests.

\section{Additional information}

Supplementary Information The online version contains supplementary material available at https://doi. org/10.1038/s41598-021-81619-4.

Correspondence and requests for materials should be addressed to D.W.

Reprints and permissions information is available at www.nature.com/reprints.

Publisher's note Springer Nature remains neutral with regard to jurisdictional claims in published maps and institutional affiliations.

(c) (i) Open Access This article is licensed under a Creative Commons Attribution 4.0 International License, which permits use, sharing, adaptation, distribution and reproduction in any medium or format, as long as you give appropriate credit to the original author(s) and the source, provide a link to the Creative Commons licence, and indicate if changes were made. The images or other third party material in this article are included in the article's Creative Commons licence, unless indicated otherwise in a credit line to the material. If material is not included in the article's Creative Commons licence and your intended use is not permitted by statutory regulation or exceeds the permitted use, you will need to obtain permission directly from the copyright holder. To view a copy of this licence, visit http://creativecommons.org/licenses/by/4.0/.

(c) The Author(s) 2021 